\title{
Side effects of electrochemotherapy in cats with squamous cell carcinoma: a retrospective study
}

\author{
Ana Rita Zeferino ${ }^{1,2}$, Joaquim Henriques ${ }^{1}$, Ana Faustino-Rocha ${ }^{2,3}$, Ana Rita Serras ${ }^{2}$ \\ ${ }^{1}$ Hospital Veterinário de Berna, Onevet group, Lisbon, Portugal. ${ }^{2}$ Faculdade de Medicina Veterinária da Universidade Lusófona de Humanidades e \\ Tecnologias, Lisbon, Portugal. ${ }^{3}$ Department of Zootechnics, School of Sciences and Technology, University of Évora, Ėvora, Portugal
}

Eletrochemotherapy (EQT) is an emerging therapeutic modality in Veterinary Oncology that combines the intravenous (IV) or intratumoral (IT) administration of chemotherapeutic agents with the application of electrical pulses ${ }^{(1)}$.

This therapy has been described to induce significant cancer remission in cutaneous squamous cell carcinoma (SCC) and a reduction of chemotherapeutic dosages, maximizing intracellular concentration of these drugs as well as reducing its systemic side effects ${ }^{(2,3)}$.

\section{Objectives}

The aim of this study was to describe the side effects observed in cats with a cytological or histopathological diagnosis of oral or cutaneous squamous cell carcinoma (SCC) of the head submitted to EQT as a primary treatment.

\section{Methods}

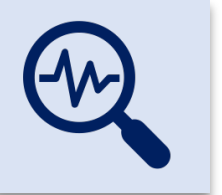

The medical records of cats treated with EQT for SCC at the "Onevet Group - Hospital Veterinário de Berna" between December 2015 and February 2018 were reviewed.

Cats with a cytologic and/or histopathologic diagnosis of head SCC (cutaneous and oral) that received at least one session of EQT were eligible for inclusion.

Cats with incomplete clinical record or without a cyto/histopathological report were excluded.

Side effects data were retrospectively classified using the Common Terminology Criteria for Adverse Events Following Chemotherapy or Biological Antineoplastic Therapy in Dogs and Cats v1.1 (VCOG- CTCAE) ${ }^{(4)}$.

\section{Results}

- Seventeen cats were treated for a total of 28 EQT sessions; The number of treatments per cat were: 1 session $(52.9 \%) ; 2(35.3 \%) ; 3(5.9 \%)$ and 4 sessions $(5.9 \%)$.

- SCC lesions treated were localized on the nasal planum $(70.6 \%)$, eyelid $(11.8 \%)$, oral $(5.9 \%)$, both on the pinna and eyelid $(5.9 \%)$ and on the pinna and face $(5.9 \%)$.

- EQT treatments were performed using bleomycin in all cases (94\% IV and 6\% IT).

- Adverse events reported per cat and per total of treatments, are described in Figure 1 (top and bottom, respectively).

- All cats had a reported adverse event at least once;

- Pain and soft tissue necrosis were present in all cats.

- The percentage of severity of adverse events are described in Figure 2. Ninety six percent of adverse events reported were grade I to III.

\section{Severity of Adverse Events}

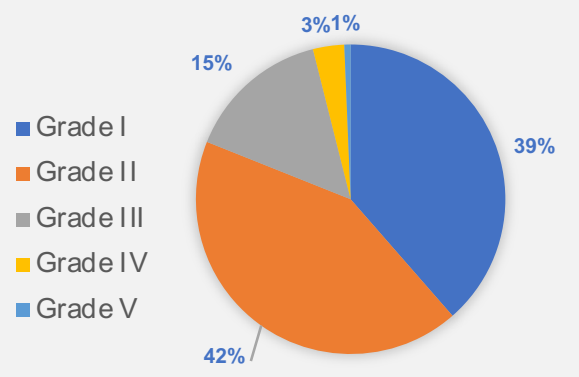

Figure 2 - Percentage of Severity of Adverse Events according to VCOG-CTCAE4.

\section{Discussion/Conclusion}

Pain, soft tissue necrosis and constitutional side effects were the most frequent adverse events, reported in more than $70 \%$ of cats included in this study. Most side effects were grade I to III according to VCOG-CTCAE and tolerable. This is comparable with previous studies of EQT in cats ${ }^{(1,5,6)}$.

Limitations of this study include its retrospective nature, small number of cases and inclusion of different locations of SCC within the head.

Further larger prospective studies accessing clinical response and side effects are warranted to promote a more conscious application of EQT. It would also be interesting to include in those future studies scales to measure the impact of EQT in cats Quality of life. 